\title{
Reasons for snack food choice and the prevalence of fruit snacking in Northern Ireland
}

\author{
R. McGill and K. M. Appleton \\ School of Psychology, Queen's University Belfast, Belfast BT9 5BP, UK
}

Previous research suggests that snacking has become a major part of modern eating behaviours and the amount that individuals do snack will continue in the future ${ }^{(1)}$. There is a multitude of reasons why individuals snack ${ }^{(2)}$. The benefits of fruit for human health are widely documented $^{(3)}$, and they seem to be a viable alternative for a snack choice; however, it is not the case. Fruit may not be perceived as much of a snack as certain 'junk foods', possibly because it doesn't meet some of the general public's snack criteria ${ }^{(4)}$.

The present exploratory study involved a diet-diary questionnaire being distributed to 200 (seventy-six male, 124 female) participants in a variety of different settings and organisations. Participants were instructed to complete the diet diary when they were just about to consume a snack food, in their own time, and then return it to the experimenter (data of which were collected for both weekend and weekday). This procedure required participants to state the snack that was about to be consumed, along with the main reason for this choice and if the snack was not a fruit then the reason why it was not a fruit. Some demographic variables were measured on the diet diary: age; occupation; diet status; deprivation score of residence, based on postcode.

The three most common snack foods consumed in the sample were: a savoury snack (crisps, breadsticks etc. $-22 \%$ ); a biscuit (19.5\%); confectionery (sweets and chocolate $-17 \%$ ). Pleasure was the most frequently given main reason for snack choice (14\%), followed by hunger $(11 \%)$ and availability and convenience $(10 \%)$. Of those who did not have fruit as their snack (173 individuals), pleasure was the most frequently given reason $(22.4 \%)$, followed by availability $(20.1 \%)$ and convenience $(7.3 \%)$. There was a significant difference in the main reason for snack choice between those who did and those who did not have fruit as their snack $\left(\chi^{2} 30.61, P=0.045\right)$. Convenience was mentioned the most often by men as their main reason for snack choice ( $14.4 \%$ of men), while pleasure was the reason mentioned most often for females ( $15.3 \%$ of females). Convenience was also the main reason that was mentioned the most often by those between 26 and 40 years of age (14.6\% of this group). There was no difference in snack choice across all levels of social deprivation. In logistic regression analyses looking at each predictor variable individually, diet (W 5.95, df $1, P=0.025$ ) and day (W 4.85, df $1, P=0.028$ ) were significant predictors of fruit consumption as a snack.

The gender difference may be reflective of gender roles in Western society, with men probably being less familiar with the preparation of food than females. For those aged 26-40 years it is likely that they will mostly be working full time and will probably base their need for convenience of a snack around their busy lifestyle. Since there was no difference in snack choice across all levels of social deprivation, it can be assumed that cost is not a major factor in snack choices in the developed world (also since it did not feature as a frequentlymentioned reason). Future research must focus on the reasons found in the present study, as highlighted by the fact that individuals are choosing fruit as a snack for different reasons than those who are not choosing fruit as a snack.

1. De Graff C (2006) Appetite 47, 18-23.

2. Jack FR, Piacentini MG \& Schroder MJA (1998) Appetite 30, 139-149.

3. Steinmetz KA (1996) J Am Diet Assoc 96, 1027-1039.

4. Jack FR, ONeill J, Piacentini MG et al. (1997) Food Qual Preference 8, 175-182. 